\title{
Germanica
}

\section{Écritures renouvelées : l'humour dans les jeunes littératures de langue allemande}

Der Humor in der jungen deutschen Literatur.

\section{Françoise Barthélémy-Toraille}

\section{(2) OpenEdition}

\section{Journals}

Édition électronique

URL : http://journals.openedition.org/germanica/321

DOI : 10.4000/germanica.321

ISSN : 2107-0784

\section{Éditeur}

Université de Lille

\section{Édition imprimée}

Date de publication : 1 décembre 2006

Pagination : 13-25

ISBN : 2-913857-18-3

ISSN : 0984-2632

\section{Référence électronique}

Françoise Barthélémy-Toraille, «Écritures renouvelées : l'humour dans les jeunes littératures de langue allemande », Germanica [En ligne], 39 | 2006, mis en ligne le 19 février 2010, consulté le 06 octobre 2020. URL : http://journals.openedition.org/germanica/321 ; DOI : https://doi.org/10.4000/ germanica.321

Ce document a été généré automatiquement le 6 octobre 2020 .

(C) Tous droits réservés 


\section{Écritures renouvelées : l'humour dans les jeunes littératures de langue allemande}

Der Humor in der jungen deutschen Literatur.

Françoise Barthélémy-Toraille

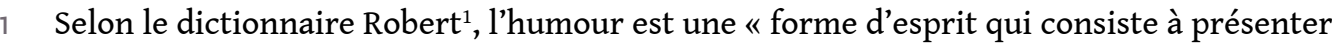
ou à déformer la réalité de manière à en dégager les aspects plaisants ou insolites ». C'est dans ce sens que nous étudierons les diverses expressions de l'humour dans la littérature allemande récente. Nous préférerons cette définition à celle que nous propose le dictionnaire Duden ${ }^{2}$ :

Gabe eines Menschen, der Schwierigkeit der Welt und der Menschen, den Schwierigkeiten und Mißgeschicken des Alltags mit heiterer Gelassenheit zu begegnen; sprachliche, künstlerische o. ä. Äußerung einer von Humor bestimmten Geisteshaltung, Wesensart.

Qualité dont est doué celui qui affronte la complexité du monde et des hommes, les difficultés et déboires du quotidien, avec équanimité ; expression langagière ou artistique par exemple d'une forme d'esprit, d'un caractère marqué par l'humour.

2 La définition française souligne qu'il s'agit d'une manière de présenter les choses susceptible d'en faire ressortir « les aspects plaisants ou insolites », et qui donc prêtent à sourire (ce qui n'exclut pas que ces aspects puissent relever également d'une certaine normalité, qui ce faisant, considérée à travers la «forme d'esprit» qui caractérise l'humour, prend un relief spécifique). À travers l'humour, ce sont des modes de fonctionnement d'une société qui peuvent être remis en question. La définition allemande proposée donne en revanche place assez fortement à ce que Kleist qualifie de "caractère imparfait du monde " ${ }^{3}$. Le Duden insiste en effet sur les problèmes et difficultés qui découlent de la nature du monde, de la nature humaine, des difficultés et revers du quotidien, aspect qui n'apparait pas dans la définition du Robert...

Ceci nous amène à poser l'hypothèse qu'en littérature, l'expression de l'humour naît d'un regard particulier porté par l'auteur sur le sujet de son écriture (personnages, 
événements, contexte, cadre du récit etc.). Ce regard peut aboutir à une "déformation " prenant souvent la forme d'une amplification, d'une exagération - qui s'apparente ici à la démarche du caricaturiste. L'auteur vise un effet à travers la réception du texte écrit, il cherche à construire une connivence avec son lecteur reposant sur ce que les définitions mentionnées ci-dessus, française comme allemande, qualifient de « forme d'esprit », voire de « don ».

Nous allons nous attacher, dans l'étude qui suit, à présenter les manifestations de l'humour et à tenter d'en interpréter le "pourquoi » à travers des textes parus récemment sous la plume d'auteurs de langue allemande, jeunes ou moins jeunes. Nous voudrions ainsi montrer que l'humour est un ressort efficace des écritures actuelles en langue allemande.

5 Nous nous pencherons d'abord sur des textes dont l'ancrage même indique la tonalité, des textes visant une forme de critique sociale et dans lesquels l'expression de l'humour, en grossissant le trait à l'instar de la caricature, met en lumière des phénomènes de mode, des phénomènes de société que les écrivains cherchent à tourner en dérision, voire à dénoncer. La critique sociale y est souvent indirecte - elle est en effet rarement l'objectif premier de ces auteurs - mais cependant efficace.

Il s'agit parfois de situations légèrement décalées par rapport à la vie « réelle » et dont la présentation, la mise en relief qui aboutit à l'humour affirmé ou sous-jacent, soulignent ce caractère. Le lecteur entre, au sens étymologique du terme, en "sympathie " avec les personnages, et les considère, dans une sorte de connivence, avec un regard proche de celui que porte l'auteur sur eux. Il en est ainsi chez Tanja Dückers, dans le roman Spielzone ${ }^{4}$ (titre qui évoque entre autres le jeu d'échec, l'échiquier comme "terrain du jeu ", et fait référence aux relations qui s'établissent dans ce roman entre les personnages, jouant entre eux des parties qui relèvent d'une série d'éléments dont le hasard n'est pas le moindre). Ces personnages sont tout sauf "romanesques ", ils vivent au quotidien dans le Berlin d'après la réunification. Parmi eux, une vieille dame, Rosemarie Minzlin, pour qui la tombe de Gerd, son mari, est un but de promenade régulier. Sa route y croise un jour celle de Laura, une petite adolescente pour laquelle, comme pour ses copains, ce lieu est un terrain d'aventure, nocturne en général, un endroit où les jeunes se retrouvent sans être dérangés, avec bières et coca, pizzas et cigarettes. La rencontre entre les deux personnages commence sur un sourire : la vieille dame et l'adolescente portent, comme en signe de ralliement, la même blouse fleurie de tournesols (comme l'indique le titre du chapitre ${ }^{5}$ ) achetée par l'une et l'autre aux soldes de H\&M... S'ouvrant sur des remarques pleines de suspicion et de reproche de la vieille dame - Laura, la jeune fille, n'est-elle pas de ceux qui choisissent la tombe de son mari comme lieu de rendez-vous? - un dialogue se noue entre les deux personnages. Il est marqué d'emblée par le décalage - un des ressorts de l'humour - entre le langage « jeune » de Laura et les réponses pleines de bon sens, d'à propos, et aussi d'un humour indiscutable, de la vieille dame. Un seul exemple donnera la tonalité du chapitre, mais aussi du roman tout entier : Rosemarie Minzlin, la vieille dame, s'inquiète de savoir si l'adolescente "elle a peut-être quatorze, quinze ans » vient souvent au cimetière, à quoi Laura répond: «Oui, j'habite tout près, je viens souvent ici, histoire de me distraire, c'est un endroit calme et reposant ». La vieille dame proteste alors, évoquant les détritus qu'elle trouve autour de la tombe - canettes vides, reliefs de pizzas - qu'il n'est pas si reposant que cela de ramasser. Laura cherche 
à éviter les reproches en répondant, tactique éprouvée, à la question de Rosemarie par une autre question :

«Cette tombe, c'est la vôtre?

Eh bien, je ne suis pas encore dedans, seulement si je continue à m'esquinter le dos ici, ça sera sûrement pour bientôt, mais " et Rosemarie regarde l'adolescente en face, « mais » poursuit-elle, «c'est ma tombe, si tu veux, parce que mon mari est enterré là.

OK, OK, le prochain coup, on ira sur la tombe d'à côté, vous énervez pas » ${ }^{6}$, répond la jeune fille.

7 À ce moment précis, les deux protagonistes découvrent qu'elles portent la même blouse. Vers la fin de ce chapitre apparaît un personnage un peu inquiétant, un genre de jeune clochard qualifié de "der Typ », un type, accompagné de son chien Yesterday, un petit clébard. Il porte en tatouage sur la joue une rose "qu'on dirait tirée d'un album de poésies $"^{7}$, tatouage qualifié à la fin du chapitre de «tatouage de chewing gum $»^{8}$, et s'adresse aux deux femmes, qu'il prend pour une grand-mère accompagnée de sa petite-fille. Il leur parle de sa défunte grand-mère dont il n'a pas su préserver la tombe alors que la concession aurait dû être renouvelée ${ }^{\%} .$.

8 L'ensemble du livre de Tanja Dückers est écrit sur le ton de l'ironie douce amère, il rend compte d'une observation fine de la réalité des petites choses et des relations qui s'établissent de manière imprévue entre les individus, ou ne s'établissent pas, laissant chacun dans sa solitude. L'écriture, en parodiant les niveaux et styles de langage correspondant aux différents personnages, souligne ainsi l'effet de contraste et le caractère improbable de ces rencontres, et donne à ces portraits, qui sont autant de miniatures, une force expressive particulière.

On retrouve à certains égards une démarche analogue - que l'on peut cependant juger moins aboutie - chez Elke Naters, dont les deux premiers romans, Les Reines et Mensonges ont été traduits en français ${ }^{10}$. Les différents idiomes en vogue, mais aussi les phénomènes de société et de mode, les relations superficielles qui caractérisent certains milieux « branchés », copains, copines, amitiés, brouilles et rivalités, les objets cultes, chaussures Prada et sacs Gucci, et à nouveau H\&M, les fringues bon marché, l'usage souvent immodéré et incontrôlé de la carte de crédit, ou le retour à la nature et les désillusions qui peuvent l'accompagner, sont vus chez Elke Naters à travers le prisme de l'humour et de la dérision : ainsi ces vacances familiales se transformant en cauchemar sous la pluie et dans l'inconfort d'une location rustique sur la côte suédoise envahie par les moustiques ${ }^{11}$...

Autre jeune auteur dont l'humour est un des traits caractéristiques, Till Müller Klug unit un regard incisif à un sens de la langue aiguisé qui nourrit son écriture de jeux sur les mots et la langue. Ainsi, dans des textes rassemblés et dits avec accompagnement musical par l'auteur dans un livre-CD, Die sprechende Droge $e^{12}$, «la drogue qui parle ", l'auteur tourne en dérision des aspects connus de tous ses lecteurs ou auditeurs de la vie moderne, allemande en particulier : le respect du piéton allemand pour le "petit bonhomme rouge », dans Die sado-masoschistische Ampel ${ }^{13}$, «Le feu rouge sado-maso »; l'amour de la voiture et de la vitesse, l'accident qui aurait pu être fatal dans Dein Freund der Baum ${ }^{14}$ "Ton ami l'arbre ", le rituel de la rupture à travers un cocasse "thème et variation » sur « changer de vie », qui s'ouvre sur « Ta nouvelle vie s'appelle Vera. Ton ancienne vie s'appelle Gabi $»^{15}$.

Le regard d'écrivains venus d'ailleurs aboutit lui aussi à un effet d'humour né du regard distancié, naïf ou plutôt faussement naïf, que portent des auteurs sur leurs 
personnages. Ce procédé peut être rattaché à la longue tradition inaugurée dans la littérature française par le «Comment peut-on être persan? » de Montesquieu dans Les Lettres persanes ${ }^{16}$. Le choc des cultures et les situations paradoxales ou cocasses qui en résultent s'expriment par exemple dans les brèves notations de Dilek Güngör, qui rassemble sous le titre Unter uns ${ }^{17}$, Entre nous, des articles et chroniques écrits pour Die Berliner Zeitung. On y découvre une famille turque qui sans doute ressemble à s'y méprendre à celle de l'auteur où à des familles proches : un père, une mère, deux sœurs, des grands-parents restés en Turquie et qui ne comprennent pas que leur fils, depuis plus de trente ans, vive en Allemagne. Tous ces personnages rencontrent au quotidien les contradictions et tensions d'une vie entre les cultures... Il y a aussi dans cette famille la tante Hatice et l'oncle Ömer, pour qui l'ours en peluche-bouillotte offert à sa petite-nièce ne peut pas s'appeler Tarik, mais a besoin d'un « nom allemand, solide, robuste " puisqu'il est un des exemples de la qualité indiscutable des produits "made in Germany $~^{18}$. La famille fête "tous les ans » Noël et la Saint-Nicolas, même si boules et guirlandes sont parfois accrochées au ficus qui orne le salon ${ }^{19}$. Télé, magnétoscope, poupées barbies pour les fillettes, graines de pistache et thé à la menthe constituent le cadre de ces courts récits où les deux mondes, celui des origines et celui du quotidien, se télescopent et coexistent.

Dans Russendisko entre autres, mais aussi dans Schönhauser Allee ${ }^{20}$, Wladimir Kaminer, venu de l'URSS disparue et installé dans le Berlin de l'après-mur, jette sur le monde qui l'entoure - en particulier le quartier de Schönhauser Allee, à Berlin - le regard de celui qui vient d'ailleurs. La naïveté vraie ou feinte dont sont empreints ces récits met en relief ce que l'habitude peut empêcher de voir: les conséquences de la chute du Mur sont présentées à travers le prisme déformant de l'humour et de la dérision, par exemple, dans un bref chapitre intitulé Der Radiojurist, Le juriste radiophonique. Nous citerons le début de ce chapitre, qui en donne la tonalité, et celle de l'écriture de Kaminer de façon plus générale.

«Il n'y avait pas eu autant de Russes à Berlin depuis 1945, disait récemment ma voisine, Madame Moll, une dame très comme il faut et aussi vieille que la Schönhauser Allee. Et pourtant, Madame Moll n'est pas plus berlinoise de naissance que nous autres. Elle est venue ici juste avant la guerre, après avoir quitté son petit village du Mecklembourg. Les Russes sont venus après Gorbatschow, a-t-elle ajouté $»^{21}$.

Mentionnons aussi, en raison d'une démarche comparable, Yoko Tawada, femme poète et écrivain japonaise qui écrit dans les deux langues, qu'elle fait parfois alterner dans le même volume. Le regard qu'elle porte sur l'Occident et sur l'Allemagne en particulier, à travers le sourire que suscitent certains de ses commentaires et remarques, est lui aussi nourri d'un humour qui lui permet d'interroger le monde dans lequel elle a choisi de vivre - et d'amener ses lecteurs à l'interroger eux aussi. Le récit titre du recueil Talisman $^{22}$ est exemplaire de cette démarche. La narratrice ne comprend pas à quoi correspond «ce bout de métal » que de nombreuses femmes, dans la ville où elle est arrivée, portent au lobe de l'oreille. Il n'est pas correct, dit-on dans le guide qu'elle a acheté dans son pays avant de gagner l'Europe, de poser aux Européens que l'on ne connaît pas bien des questions ayant directement trait à leur corps ou à leur religion. La narratrice émet donc, sans pouvoir la vérifier, l'hypothèse qu'il s'agit-là d'un talisman, ce qui l'amène à une série d'autres questions: cette ville est-elle donc si dangereuse ? Et pourquoi le talisman est-il surtout porté par des femmes? Lorsqu'enfin elle connaît suffisamment bien une étudiante vivant dans le même foyer qu'elle et 
l'interroge, la réponse de Gilda ne fait que la renforcer dans son idée, car la simplicité de la réponse ne peut être qu'une feinte. Aussi quand plus tard Gilda, dont l'ordinateur semble attaqué par un virus, qualifié par la narratrice de "force mauvaise», colle dessus des autocollants proclamant «Non merci!» avec une auto, une centrale atomique, une arme comme illustration, la narratrice en conclut que son amie a suivi son conseil et s'est procuré des talismans, même si ceux qu'elle a choisis ne correspondent pas à ce qu'elle-même aurait attendu. Elle s'étonne d'ailleurs de la politesse à ses yeux excessive qui s'exprime à travers ce «Non merci ! ${ }^{23}$. La narratrice ne comprend d'ailleurs plus vraiment ce qui se passe quand Gilda colle les mêmes talismans sur son vélo, sur son réfrigérateur et sur la porte de son appartement... Les interprétations que construit la narratrice de scènes de sa nouvelle vie sont élaborées en référence avec les coutumes de son pays d'origine: ainsi quand Gilda décide de suivre un régime, la narratrice y voit une cérémonie de purification et constate avec étonnement que « dans cette ville, le jour, l'heure, la prière qui ouvre la cérémonie ne sont pas fixés $»^{24}$. Cette démarche de l'écriture, qui articule le récit autour de l'humour né du regard « venu d'ailleurs » s'avère d'une particulière efficacité pour faire ressentir les effets de l'étranger, voisin de l'étrange. Le chapitre que nous évoquions se conclut sur un constat un peu amer par rapport à la relation de la narratrice à son amie Gilda, et plus généralement à la familiarité qu'elle croyait avoir acquise avec le monde dans lequel elle vit désormais: "Je ravalais les mots que je voulais lui dire, car j'eus l'impression soudain que c'était une étrangère qui - et pourtant je vis dans sa langue ne me comprenait pas $»^{25}$.

15 L'humour n'est cependant pas l'apanage exclusif des jeunes écrivains, chez qui les aspects que nous venons de citer peuvent sembler parfois relever d'une certaine attitude ludique. Il est présent également dans des textes dus à la plume d'écrivains appartenant à un paysage littéraire allemand plus traditionnel. Le tout premier d'entre eux, le prix Nobel Günter Grass, pratique une écriture volontiers caustique que l'humour a nourrie dès ses tous premiers romans. Si nous nous penchons sur l'un des derniers, Toute une histoire ${ }^{26}$, force est de constater qu'une étude de l'humour dans ce roman dépasserait le cadre de cet article. Nous nous contenterons ici de saluer les deux silhouettes évoquant le couple Don Quichotte et Sancho Pança, telles qu'elles apparaissent entre autres au livre cinq du roman de Günter Grass, perdues dans leurs vastes manteaux et penchées au bord de l'abîme qui surplombe une mine de lignite à ciel ouvert abandonnée, sous un ciel bas et sous l'abri dérisoire d'un parapluie de poche, un Knirps, tenu par le plus grand des deux personnages ${ }^{27}$...

Dans le roman intitulé Wäldschestag ${ }^{28}$, par référence à la fête qui se déroule traditionnellement le mardi de la Pentecôte et se transforme en une gigantesque beuverie, dans la région qui sert de cadre à son roman, Andreas Maier brosse une fresque évoquant, souvent avec un humour grinçant, les relations complexes et aux multiples facettes nouées entre les membres d'une communauté villageoise, voisins ou parents, dans une bourgade de Hesse. Quelques scènes seront ici mentionnées rapidement : le roman s'ouvre sur l'enterrement du vieil Adomeit, qui en est le grand absent et le personnage principal. La tonalité de l'ensemble est donnée dès les premières pages: une ironie grinçante par laquelle Maier touche droit au but, dénonçant l'hypocrisie de relations sociales de façade, ainsi le dévouement apparent de la belle-fille pour son beau-père malade, ou les conversations entre voisins lors des obsèques qui viennent perturber les festivités et festins de la Pentecôte, auxquelles les propos pleins d'onction du prêtre offrent un contrepoint cocasse. Un des points 
culminants du roman est atteint avec l'ouverture du testament par lequel le défunt laisse tous ses biens à sa gouvernante, une vieille femme noyée dans l'alcool, au grand dam de sa famille venue de loin spécialement pour l'occasion, fils, belle-fille, petite-fille et son soupirant éconduit, flanqués d'une extraordinaire vieille tante pour qui «le Service du travail du Reich avait été le plus beau moment de sa vie ${ }^{29}$. La tante Lenchen répète cela tout au long du roman comme un leitmotiv, jouant sur l'irritation de ses proches qui tentent toujours de la faire taire quand elle s'aventure ainsi vers des propos politiquement fort incorrects... Le personnage principal lui-même, le vieil Adomeit, que le lecteur découvre en particulier à travers les témoignages des autres, vieux fou solitaire et empêcheur de danser en rond, est doté d'un humour féroce dont les nombreux exemples proposés tracent peu à peu le portrait d'un personnage au caractère caustique et indomptable. Ses obsèques et les dispositions testamentaires qui les accompagnent sont comme un dernier pied de nez à l'hypocrisie du monde dans lequel il a vécu.

17 Nous touchons ainsi à cette forme particulière de l'humour noir qualifié en allemand d'humour de gibet, « Galgenhumor », largement représentée dans l'écriture allemande contemporaine. Les degrés de "noirceur» sont divers, et nous allons en évoquer quelques aspects.

Dans Selige Zeiten, brüchige Welt, publié en français sous le titre La pitoyable histoire de Leo Singer ${ }^{30}$, Robert Menasse construit une relation mère-fils symboliquement parallèle à cet amour-haine (Haßliebe) si fréquent dans la littérature autrichienne, qu'il présente à travers des anecdotes grinçantes - les cadeaux d'anniversaire du fils Leo, gants et écharpes en laine grossière proviennent directement du stock de l'entreprise de confection dirigée par son père et sont emballés par sa mère dans un papier qu'elle récupère et plie soigneusement pour la prochaine occasion. L'auteur souligne par la tonalité de son récit l'hypocrisie des fêtes de Noël et du sapin dans cette famille juive " assimilée ", thématique présentée de manière plus souriante par Dilek Güngör, nous l'avons vu... Le sommet absolu est atteint dans un humour noir qu'on croit accompagné d'un rire sardonique, quand le fils, fidèle malgré lui aux dernières volontés de sa mère, décédée en Autriche, emporte ses cendres en Argentine où la famille avait fui les nazis et part les disperser en une zone désertique. Après plusieurs centaines de kilomètres parcourus seul en voiture, l'urne à côté de lui sur le siège avant, au lieu souhaité par sa mère, il disperse les cendres et ne peut empêcher les larmes de lui monter aux yeux. Or il a commis l'erreur fatale de vider l'urne face au vent, et les cendres de sa mère mêlées à ses larmes viennent se coller sur son visage. La grandeur macabre de la scène constitue un sommet de l'humour noir et grinçant dans ce roman de l'échec, thématique soulignée par l'adjectif «pitoyable » utilisé dans le titre de la traduction française...

19 Le roman de Urs Widmer, Le Livre de mon père ${ }^{31}$, accumule les scènes qui suscitent le sourire, ou même le rire, pour construire un portrait tendre et douloureux d'une relation père-fils dans un couple difficile, où la distance s'accroît peu à peu entre les deux parents, jusqu'à la scène finale qui suit immédiatement le décès solitaire du père : la mère emplit sac après sac de papiers amassés par le père, sans laisser au fils le temps de sauver au moins "le livre du père $~^{32}$, titre en allemand de ce roman, livre écrit au jour le jour par le père et que le fils a réécrit, à travers le livre que le lecteur est en train de refermer - à l'instar du lecteur de À la recherche du temps perdu. De nombreux passages traduisent à travers une pointe d'humour la complexe relation entre les 
personnages. Nous citerons à titre d'exemple le chapitre consacré aux amours du père, qui parallèlement permet au narrateur d'évoquer le séjour de ce dernier à Paris. S'ouvrant sur le constat, qui situe la tonalité générale du passage, qu' "un fils est toujours certain que son père n'a jamais couché avec une femme ; à la rigueur avec celle qui est devenue sa mère, ou tout au plus cette unique fois $\star^{33}$, le narrateur énumère toutes celles pour qui son père a soupiré, construisant une litanie «la fille aux taches de rousseur... $»^{34}$ suivie par " une Regula, qu'il idolâtra de loin », "une Marie-Jo qu'il allait chercher à l'école", "une Stéphanie lui expliqua la carte du ciel», "une Susanne ». Le destin ultérieur de chacune est résumé en une lapidaire parenthèse qui suit le récit d'ébats avortés ou d'entreprises de séduction ratées par le père. Ces énumérations cocasses culminent dans l'évocation de trois d'entre elles avec qui le père se rend en particulier au cinéma :

Ce qu'il préférait encore, c'était d'emmener ses femmes dans les nouveaux cinémas. [...] Quand Charlie Chaplin fourrait dans un bec de gaz le crâne du gros policier stupide, ils hurlaient de rire, lui et Paula et Sophie et Renate et se cramponnaient les uns aux autres, les larmes aux yeux. Il vit huit fois Le Cuirassé Potemkine, trois fois avec Renate, deux fois avec Sophie, une fois avec Paula - elle trouva le film ennuyeux - et deux fois seul ${ }^{35}$.

Le cœur de ce chapitre narre les déboires du père avec Hélène, une jeune assistante de français de son université qu'il finit par perdre, suivre jusqu'à Paris, où il la retrouve pour la reperdre à nouveau. On y apprend que la jeune femme « connaissait toujours les derniers ragots de son pays. Que le glorieux et plus que grisonnant Pétain confiait depuis peu son bâton de maréchal à une maîtresse d'à peine vingt ans, ou qu'Anatole France était maintenant si sentencieux qu'il ne parlait plus qu'en majuscules. Que Paul Claudel voulait convertir André Gide... $»^{36} \mathrm{Et}$ lorsque excédée de ses attentions la jeune femme finit par lui enjoindre, au jardin du Luxembourg, de « débarrasser le plancher, sinon il allait y avoir un malheur », le jeune homme s'éloigne, honteux et confus sous les yeux des badauds :

[il] tourna les talons et - aussi dignement qu'il put - se dirigea vers la sortie. Il avait parcouru déjà une dizaine de mètres lorsqu'il s'avisa qu'il avait oublié de saluer en ôtant son chapeau. Il l'ôta donc, aussi parce qu'il lui fallait essuyer son front couvert de sueur ${ }^{37}$.

21 Ce passage rend parfaitement l'esprit dans lequel est écrit tout le livre, nourri de notations parfois minimalistes qui confèrent à l'ensemble une présence, une immédiateté mais aussi parfois une dimension surréelle - on peut interpréter ainsi l'accumulation des prénoms et des échecs féminins qu'ils symbolisent dans le passage présenté. C'est encore plus vrai si l'on évoque une des scènes d'ouverture du roman, au cours de laquelle le père, alors âgé de douze ans, lors d'une sorte de cérémonie initiatique, doit parcourir seul, sous l'orage et la grêle, un trajet difficile qui le mène de la maison de ses parents, en ville, à leur village natal ou l'assemblée des villageois l'attend dans l'église. L'enfant est passé auparavant auprès des cercueils empilés devant chacune des maisons : "Chaque habitant du village recevait à sa naissance un cercueil où on le coucherait quand le temps serait venu. Jusque-là, le cercueil attendait devant la maison $\aleph^{38}$. À cette scène d'ouverture - précédée d'ailleurs du récit qui reviendra à la fin du roman, celui de la mort du père, où le grotesque rejoint et colore le tragique répond en contre-chant la fin du roman, lorsque dans une $2 \mathrm{CV}$ poussive le fils part chercher le cercueil du père. Il apprend alors que les cercueils sont devenus bois de chauffage ${ }^{39}$ de la bouche d'un laconique tenancier de bistrot qui s'avère être un cousin - 
mais lui réclame tout de même, quand il s'éloigne le paiement de la bière qu'il lui a servie...

\section{NOTES}

1. Dictionnaire alphabétique et analogique de la langue française, éd. Le Robert, 6 volumes, 1983.

2. Duden, das große Wörterbuch der deutschen Sprache in 8 Bänden, Dudenverlag, 1994.

3. Heinrich von Kleist, Die Marquise von $O$, in : Heinrich von Kleist, sämtliche Werke und Briefe, hrsg. von Helmut Sembdner, Carl Hanser Verlag, München, 1964, Bd 2 : il y est mentionné en conclusion du récit l'ordre vicié du monde et son caractère imparfait et fragile, qualifié de " gebrechliche Einrichtung der Welt », p. 143.

4. Tanja Dückers, Spielzone, Aufbau Taschenbuch Verlag, 2000.

5. Ib., pp. 53-68 : Sonnenblumenblusen.

6. Id., p. 56: «'Bist du öfter hier?' fragt Rosemarie das Mädchen, das vielleicht vierzehn, fünfzehn Jahre alt ist, argwöhnisch. [...]

'Ja, ich wohne gleich da in der Thomasstraße, bin oft hier... zum Zeitvertreib [...] das hier ist so'ne Art Ruhestätte...' jetzt muß das Mädchen lachen, 'ich meine Erholungsort oder wie soll ich sagen... für mich'

'Aber so erholsam ist das für andere nicht, hier Coladosen und alte Pizzen aufzulesen.'

‘Äh, sorry, aber die Mülls sind immer voll mit Blumenzeugs und so... Ist das Ihr Grab hier, ja?'

'Na, noch bin ich da nicht drin, aber wenn ich mir weiter den Rücken so verbiegen muß, bestimmt bald' Rosemarie faßt das Mädchen ins Auge, 'aber', fährt sie fort, 'es ist, wenn du so willst, mein Grab, denn mein Mann liegt dort begraben.'

'Okay, okay, nächstes Mal gehen wir ein Grab weiter, und nun regen Sie sich mal ab.' Sagt das Mädchen und will aufstehen. » 
7. Id., p.63: «Auf seiner linken Wange die kleine tätowierte Rose, eine richtige Poesiealbumrose. »

8. Id., p. 68 : «Wie niedlich, ein Mann mit einem Gesicht wie ein Kamikaze-Flieger, der sich Kaugummi-Tattoos aufklebt.»

9. Id., p. 66 : « Und ihr... ihr geht zusammen zum Einkaufen, ja ? Oma und Enkelin, ist ja niedlich. Meine Großmutter war auch hier auf dem Friedhof, Tag und Nacht meine ich, aber ich konnte das Familiengrab nicht mehr bezahlen [...] und jetzt gibt's das Grab nicht mehr... war ein schönes Grab. »

10. Elke Naters, Königinnen, Kiepenheuer \& Witsch, 1998, traduit sous le titre Les Reines, traduction Françoise Toraille, Denoël, 2000 ; Lügen, Kiepenheuer \& Witsch, Mensonges, traduction Florence Tenenbaum, Denoël, 2001.

11. Ib., p. 120 : «So ein scheißwindiges verzecktes saublödes Scheißschweden. Viecher, wo man liegt und sitzt. Auf dem Scheißhaus haben sich die Hornissen eingerichtet, die Spinnen rennen über die Betten. Mücken sowieso und Ameisen. So eine Natur will ich nicht. Ich will eine insektenfreie Natur, in der ein paar Bienchen umhersummen und viele bunte Schmetterlinge. »

12. Till Müller-Klug, Die sprechende Droge, slam poetry, Verlag Der gesunde Menschenversand, 2000 - le nom de la maison d'édition prête lui-même à sourire...

13. Id., p. 13-14 : Die sadomasoschistische Ampel. Ce texte court s'ouvre sur la phrase suivante : « Deutschland, einziges Land der Welt, wo Fußgänger morgens um vier an einer gottverlassenen Kreuzung bei Rot stehen bleiben" et donne à ce feu rouge et à son petit bonhomme une dimension mythique.

14. Id., p. 7-12 : Dein Freund der Baum, Angewandte Landschaftslyrik.

15. Id., p. 15-18: Toller Typ. «Heute abend um elf Uhr beginnt dein neues Leben. Dein neues Leben heißt Vera. Dein altes Leben heißt Gabi. »

16. Montesquieu, Lettres persanes, publié sans nom d'auteur à Amsterdam en 1721.

17. Dilek Güngör, Unter uns, Edition Ebersbach 2004.

18. Ib., pp. 38-39 : Der deutsche Bär: «Wie soll er denn heißen? » fragt Onkel Ömer. "Tarik», sagt Selma. Onkel Ömer zieht die Augenbrauen zusammen. « Wie kommst du denn darauf? Du kannst deinen deutschen Bären doch nicht Tarik nennen », sagt er und überlegt, ob ihm nicht rasch ein solider Qualitätsname einfällt.

19. Id., Alle Jahre wieder, p. 44 « Meinem Vater liegt viel an einem schönen Weihnachtsfest. Wir sind keine Christen, aber wir feiern Weihnachten, seit ich denken kann. » L'auteur a peut-être pensé en choisissant ce titre au récit de Heinrich Böll?

20. Wladimir Kaminer, Russendisko, Manhattan Verlag, Munich, 2000, Schönhauser Allee, Manhattan Verlag, Munich, 2001, mais aussi Die Reise nach Trulala, Manhattan Verlag, Munich, 2002. Plusieurs titres de cet auteur sont disponibles en traduction française aux éditions Belfond. 21. "So viele Russen wie jetzt gab es in Berlin seit 1945 nicht mehr », sagte neulich meine Nachbarin Frau Moll, eine vornehme Dame, die genau so alt ist wie die Schönhauser Allee. Dabei ist Frau Moll keine geborene Berlinerin, genau wie wir. Kurz vor dem Krieg zog sie aus einem mecklenburgischen Dorg hierher. "Die Russen kamen nach Gorbatschow", meinte sie. Schönhauser Allee, Goldmann Manhattan 54168, p. 115.

22. Yoko Tawada, Talisman, konkursbuch, Tübingen, 1996, en particulier le récit éponyme, p. 52-57.

23. Ib., p. 55 : « Es kam mir zwar zu höflich vor, gleichzeitig eine böse Kraft abzulehnen und sich dafür zu bedanken, aber vielleicht sollte mit dem Wort Danke versucht werden, keine Agression bei dem Gegner hervorzurufen ».

24. Id., p. 56 : «In dieser Stadt ist es aber nicht festgelegt, an welchem Tag, zu welcher Uhrzeit und mit welchem Gebet man die Zeremonie beginnt. »

25. Id., p. 57 : «Ich verschluckte die Worte, die ich ihr sagen wollte, denn sie erschien mir plötzlich wie eine Fremde, die mich - obwohl ich in ihrer Sprache lebte - nicht versteht. » 
26. Günter Grass, Ein weites Feld, Steidl Verlag, 1995, traduit par Claude Porcell et Bernard Lortholary sous le titre Toute une histoire, Le Seuil, 1997.

27. Ib., p. 512, «Hier, hier und dort sehen wir sie als Paar vervielfältigt, dicht bei dicht und hintereinander gestaffelt, in Mänteln und unter Hüten, von verschiedenem Wuchs, hundert und mehr Tagundnachtschatten mit Objekt, gedrängt und gefährlich nah an den Rand geschoben; doch standen sie in solcher Anhäufung nur im Prinzip, in Wirklichkeit aber allein. Auf all das drückte ein tiefer Himmel, dessen Wolken übersättigt zu sein schienen, denn in der Ferne, wo sie als Säcke durchgingen, regnete es in Schleiern; sonst aber geschah nichts. »

28. Andreas Maier, Wäldschestag, Suhrkamp Verlag 2000, traduit par Françoise Toraille sous le titre Le mardi de la forêt, éd. Métailié 2003.

29. Id., p. 124, « Der Reichsarbeitsdienst sei ihre schönste Zeit gewesen. Alle ihre Freundinnen, die jetzt alle tot seien, habe sie im Reichsarbeitsdienst kennengelernt, und Parteilieder hätten sie auch nur in Pommern gesungen, in Thüringen hätten sie Volkslieder gesungen, und die hätten ihr übrigens auch viel besser gefallen [...] Das ganze Reich habe sie durch den Reichsarbeitsdienst kennengelernt, es sei das beste für sie gewesen [...]».

30. Robert Menasse, Selige Zeiten, brüchige Welt, Residenz Verlag, Wien, 1991, traduit par Christine Lecerf sous le titre La pitoyable histoire de Leo Singer éd. Verdier, 2000.

31. Urs Widmer, Das Buch des Vaters, Diogenes Verlag, 2004, traduit par Bernard Lortholary sous le titre Le livre de mon père, éd. Gallimard, avril 2006.

32. "'Wo ist das weiße Buch ?' rief ich. - 'Hab ich dir doch gesagt', sagte Clara, über die untere Schublade des Bigla gebückt. 'Im Müll. Gott sei Dank ist die erste Fuhre gleich abgeholt worden. [...] Allerdings, als eine halbe oder auch eine ganze Stunde später meine Mutter die Müllsäcke einen nach dem anderen zur Gartentür schleifte, ging ich doch zu ihr und half ihr. Papier ist schwer. ", Das Buch des Vaters, Diogenes 2005, p. 207.

33. Ib., p. 44 : « Jeder Sohn ist sich gewiß, daß sein Vater nie mit einer Frau geschlafen hat; kaum mit der, die dann seine Mutter wurde, oder allenfalls jenes eine Mal».

34. Le Livre de mon père, p. 49 et suivantes.

35. «Am liebsten allerdings führte er seine Frauen in jene neuen Kinos aus. [...] Wenn Charlie Chaplin den Schädel des blöden riesengroßen Polizisten in eine Gaslaterne steckte, brüllten er und Paula und Sophie und Renate vor Lachen und krallten sich aneinander, mit Tränen in den Augen. Den Panzerkreuzer Potemkin sah er achtmal, dreimal mit Renate, zweimal mit Sophie, einmal mit Paula - sie fand den Film langweilig - und zweimal allein. » Das Buch des Vaters, pp. 56-57.

36. Id., p. 49 : « Hélène wußte immer den neuesten Klatsch aus ihrer Heimat. Daß der in Ehren ergrauende Pétain seinen Marschallstab seit neuestem einer kaum zwanzigjährigen Geliebten überließ oder daß Anatole France inzwischen so altersweise war, daß er nur noch in Großbuchstaben sprach. Daß Paul Claudel André Gide zum rechten Glauben bekehren wollte. »

37. Id., p. 54 : «Spaziergänger scharten sich um sie und meinen Vater, so daß dieser 'Aber ich, Hélène', stammelte, sich umdrehte und - so gefaßt, wie er es schaffte - dem Ausgang des Parks zuschritt. Er war schon ein Dutzend Meter weit weg, als er merkte, daß er den Hut nicht gelüftet hatte. Er hob ihn also ab, auch, weil er sich den Schweiß von der Stirn wischen mußte. »

38. Le Livre de mon père, pages 24 à 49 , et en particulier p. 30 pour le passage cité ici. «Jeder im Dorf kriegte bei seiner Geburt einen Sarg, in den er dann, wenn seine Zeit gekommen war, gelegt wurde » Das Buch des Vaters, p. 24.

39. Ib., pp. 199-200 pour la traduction française, 201-202 dans l'édition allemande: «Beim Umbau, da war der Vater noch der Wirt, da haben wir das ganze Gerümpel verholzt. [...] Wir haben ein paar tausend Touristen im Jahr. Stellen Sie sich vor, Sie sind Japaner, sie machen die ganze teure Reise, Sie wollen es schön haben, und dann stolpern Sie überall über Särge. », paroles prononcées par l'aubergiste et cousin éloigné du narrateur. 
40. Das steinerne Herz, ein historischer Roman aus dem Jahre 1954, Stahlberg Verlag, Karlsruhe, 1956, Bibliothek Suhrkamp 2002, Le cœur de pierre, traduit par Claude Riehl, éd. Tristram 2002.

\section{RÉSUMÉS}

Il s'agit dans cet article de montrer que l'humour est un des ressorts efficaces des écritures actuelles en langue allemande : elles s'articulent - par la voix d'auteurs comme Tanja Dückers, Elke Naters, Till Müller-Klug, autour de l'observation des "petites choses du quotidien ». A travers des textes souvent brefs sont mis en relief des travers de la société dans laquelle vivent ces auteurs Par une écriture alerte et sans concession, ils jettent un regard décalé, mais non dénué de tendresse, sur le monde qui les entoure, établissant ainsi une connivence efficace entre eux, leurs personnages, leurs lecteurs.

Des écrivains "venus d'ailleurs" possèdent au plus haut point cette capacité d'interroger une société pour eux déroutante, dont ils remettent en question les règles en les interprétant au filtre de leur expérience première. L'humour, parfois grinçant, s'inscrit également dans des œuvres romanesques ambitieuses, de Grass à Urs Widmer ou Robert Menasse. L'humour, élément indiscutable du " plaisir de lire ", permet une remise en question, une critique des pratiques et des normes.

In diesem Artikel wird versucht, die Bedeutung des Humors in der jungen deutschen Literatur zu zeigen. Autoren wie Tanja Dückers, Elke Naters, Till Müller-Klug, geht es um die Darstellung der «kleinen Dinge des Alltags». In oft kurzen Prosatexten werden bestimmte Aspekte der zeitgenössischen Gesellschaft durch Hervorhebung, Distanzierung, durch einen kunstvoll « naiven » Blick denunziert und in Frage gestellt.

Der «Blick von Außen» ermöglicht mit besonderer Wirksamkeit diese Infragestellung des Banalen und Selbstverständlichen. Die Regel einer «fremden» Gesellschaft werden aus der Perspektive des im Herkunftsland Erlebten mit neuer Schärfe wahrgenommen. Galgenhumor als wirksame Form des Humors liegt auch anspruchsvollen Romanen, von Grass bis Menasse über Urs WIdmer oder Andreas Maier, zugrunde. Als wichtige Bedingung des Lesevergnügens führt der Humor zur gesellschaftskrititschen Stellungnahme.

\section{INDEX}

Mots-clés : humour

oeuvrecitee Das Buch des Vaters, Königinnen, Lügen, Russendisko, Schönhauser Allee, Selige Zeiten - brüchige Welt, Spielzone, Die Reise nach Trulala

\section{AUTEURS}

\section{FRANÇOISE BARTHÉLÉMY-TORAILLE}

Université de Paris 12 Val de Marne 